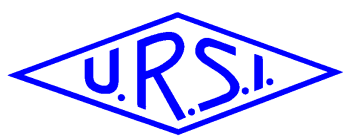

\title{
Analytical Formulation for Loop Antennas Valid from the RF to Optical Regime: A Review
}

\author{
Ryan J. Chaky* (1), Jogender Nagar ${ }^{(1)}$, Arnold F. McKinley ${ }^{(2)}$, Mario F. Pantoja ${ }^{(3)}$, and Douglas H. Werner ${ }^{(1)}$ \\ (1) Department of Electrical Engineering, The Pennsylvania State University, University Park, PA, USA \\ (2) Department of Electronic and Electrical Engineering, University College London, London, UK
}

(3) Department of Electromagnetics, University of Granada, Spain

\begin{abstract}
Loop antennas are among one of the simplest antennas to construct. Yet, despite this fact, the mathematical complexity of the associated radiation integrals has complicated the understanding and modelling of such structures. Recently, a full analytical theory for the radiation properties of loop antennas has been derived, which is able to bridge this gap and provide a basis for better understanding the behavior of these antennas across all frequency ranges. Further, these models have been extended to include the effects of loading and coupling, as well as have revealed interesting properties such as superdirectivity by enabling rapid parametric studies and optimizations to be carried out.
\end{abstract}

\section{Introduction}

In years past, the loop antenna has received less attention than other antennas such as its counterpart, the classical dipole antenna. However, with the advent of metamaterials there has been a renewed interest in understanding and utilizing loop antennas, especially in a split-ring configuration [1]. Furthermore, loop antennas have received considerable interest in the far-IR to optical frequencies for applications such as energy harvesting, biological sensing, spectroscopy, and microscopy [2]. As in the analysis of all antennas, it is necessary to first determine the current throughout the radiating structure. Due to the radially symmetric geometry of a circular loop, Storer was able to derive an analytical solution for the associated current distribution by solving the EFIE using the methods of complex Fourier series [3]. Wu recognized a convergence problem with these Fourier coefficients and amended their definition [4]. Following this work, Werner derived the radiated far fields with the current described in its most general form as a series of complex Fourier modes [5]. All of these developments assume that the frequency range of operation is in the RF regime. As a result, these methods do not account for the material dispersion inherent in metals at higher frequencies, such as in the far-IR to optical regimes. McKinley extended the work of Storer and $\mathrm{Wu}$ in [6], to account for this dispersion by introducing a complex surface impedance along the loop. Using these definitions, Werner and $\mathrm{Lu}$ et al. developed a comprehensive, analytical model of the loop antenna valid from the RF to the optical regime [7]. Later this work, in collaboration, was extended to include the effects of loading $[8,9]$ following the methods of Harrington [10] as well as mutual coupling using the induced-EMF method [11]. Furthermore, given the analytical nature of the model, thin loop antennas and their interactions can be modeled more efficiently while using much less RAM than a fullwave solver would require. This is especially true when modelling dispersive loops. The following discusses the theory, optimization, and unexpected results associated with general thin-wire loops and, in particular, nanoloops.

\section{Theory of Unloaded and Loaded Loops}

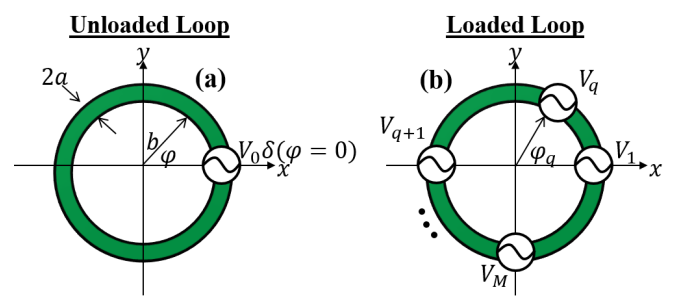

Figure 1. a) Geometry of circular loop with radius $b$ and wire radius $a$ fed by a delta-gap source at $\varphi=0$. b) Loaded geometry having identical dimensions as a) with M ports located at $\varphi=\varphi_{q}$.

The geometry of both the unloaded and loaded loops with radius $b$ and wire radius $a$ are provided in Figure 1. The loop is fed by a delta-gap voltage source located at $\varphi=0$. The thin-wire approximation is assumed such that $a \ll$ $b$ and $a \ll \lambda$. As a result, the current only varies as function of the coordinate $\varphi$ around the loop. Following the moment method, the current is decomposed into the entire-domain complex exponential basis as a Fourier series. This is described below in Equation (1). The current can further be expressed in terms of the input voltage and the modal admittances $Y_{m}^{\prime}$ as shown in Equations (2) and (3). The addition of the surface impedance term $Z_{s}$ in the modal admittance expression accounts for the material dispersion and thus extends this derivation beyond the RF regime. A further description of $Z_{s}$ is found in [6].

$$
I(\varphi)=\sum_{m=-\infty}^{\infty} I_{m}^{\prime} e^{j m \varphi}=V_{0}\left[\sum_{m=-\infty}^{\infty} Y_{m}^{\prime} e^{j m \varphi}\right]
$$




$$
\begin{gathered}
Y_{0}^{\prime}=\left[j \pi \eta_{0} a_{0}+(b / a) Z_{s}\right]^{-1} \\
Y_{m}^{\prime}=\left[j \pi \eta_{0}\left(a_{m} / 2\right)+(b / a)\left(Z_{s} / 2\right)\right]^{-1} \\
Z_{s}=\frac{\gamma}{\sigma} \frac{J_{0}(\gamma a)}{J_{1}(\gamma a)}
\end{gathered}
$$

For the case of the loaded loop with $\mathrm{M}$ discrete ports, each with a voltage source and complex load impedance, the modal current expression is modified by the inclusion of the associated series voltage drop, shown in Equation (4). Hence, it is necessary to first solve for the currents at each port in order to fully specify the modal currents. This can be accomplished by means of solving a matrix equation [8]. The far-zone electric fields are derived in [7] and presented for reference as Equation (5). The directivity, radiation and loss resistances, gain, and power can be derived using standard antenna principles [12] with analytical solutions provided in [7].

$$
\begin{gathered}
\tilde{I}(\varphi)=\sum_{q=1}^{M}\left[\sum_{m=-\infty}^{\infty} Y_{m}^{\prime} e^{j m\left(\varphi-\varphi_{q}\right)}\right]\left\{V_{q}-Z_{q} I\left(\varphi_{q}\right)\right\} \\
\tilde{E}_{\theta}=j \frac{\eta_{0} e^{-j k_{0} r} \cot \theta}{2 r} \sum_{m=1}^{\infty} m j^{m}\left\{\tilde{I}_{m} e^{j m \varphi}-\tilde{I}_{-m} e^{-j m \varphi}\right\} J_{m}(w) \\
\tilde{E}_{\varphi}=-\frac{\eta_{0} e^{-j k_{0} r} k_{b}}{2 r} \sum_{m=0}^{\infty} \epsilon_{m} j^{m}\left\{\tilde{I}_{m} e^{j m \varphi}+\tilde{I}_{-m} e^{-j m \varphi}\right\} J_{m}^{\prime}(w)
\end{gathered}
$$

\section{Coupling Between Loops}

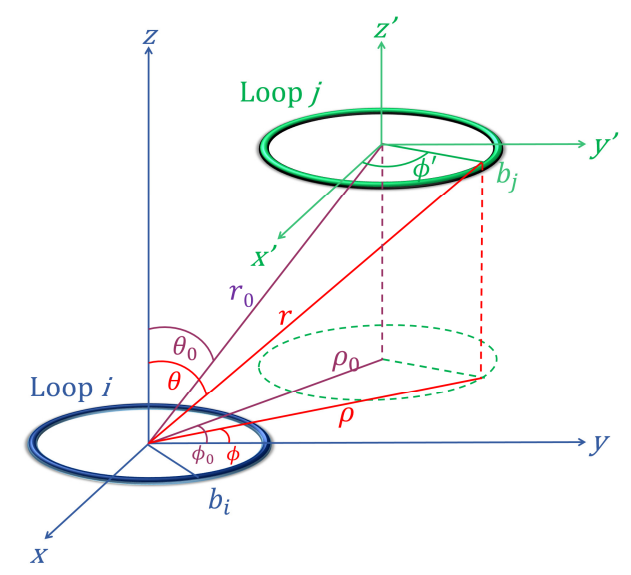

Figure 2. Coupling configuration between active loop $i$ and passive loop $j$.

The induced EMF method [12] in combination with the theory presented above can be used to model and analytically study the coupling between two or more arbitrary loops. The coupling configuration is shown in Figure 2 with a single active loop $i$ and passive loop $j$. For the sake of brevity, only the general form of the current induced on the passive loop is described below. In this expression, the $V_{i j}$ are a function of the tangentially induced field from the active loop, $Y_{p, j}^{\prime}$ are the modal admittances of the passive loop, $\Phi^{\prime}$ and $\phi^{\prime}$ are the angular coordinates around the passive and active loops respectively. All remaining derivations of the associated far fields and full analytical solutions are thoroughly discussed in [11]. If the two loops are of the same size or operating in the same mode, Equation (6) shows that the resulting induced current will be of the same form as the source current, modulated by the induced tangential field. In addition to the fully analytical approach, a pseudoanalytical formulation using the near fields can be implemented numerically. In this case, the induced current may be decomposed into its Fourier modes and Equation (4) in combination with array factor theory can be used to compute the total radiated field. This numerical implementation further extends the utility of this theory to handle an arbitrary induced excitation.

$$
I_{j}\left(\Phi^{\prime}\right)=V_{i j}\left[\sum_{p=0}^{\infty} Y_{p, j}^{\prime} \cos \left(p\left(\Phi^{\prime}-\phi^{\prime}\right)\right)\right]
$$

\section{Superdirectivity Phenomenon}

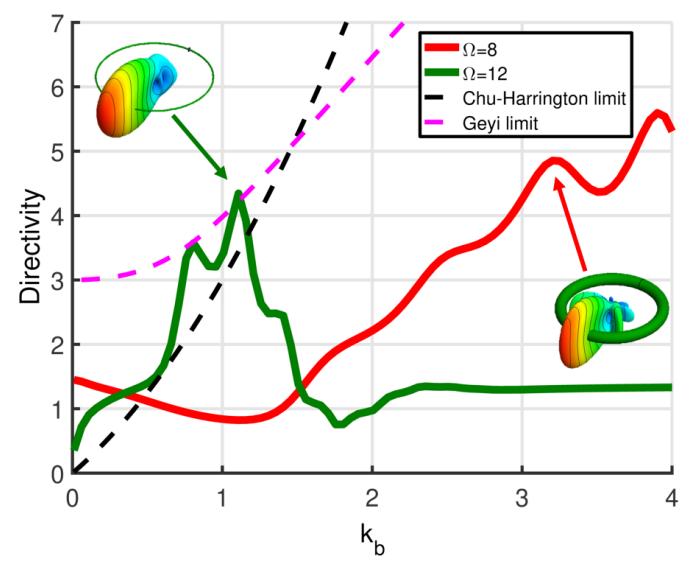

Figure 3. Frequency sweep of directivity for thin $(\Omega$ $=12)$ and thick $(\Omega=8)$ nanoloops in comparison to the Chu-Harrington and Geyi superdirective limits with insets of the full radiation pattern at two frequencies of interest.

The analytical nature of the model presented allows for rapid exploration of the possible design space. Such exploration has revealed that it is possible for a single nanoloop, in isolation, to exhibit superdirectivity [13]. Though it is shown that a thin loop $\left(\Omega=2 \ln \left(\frac{2 \pi b}{a}\right)=12\right)$ has a low radiation efficiency, a thicker loop $(\Omega=8)$ is capable of exhibiting a high gain over a wide bandwidth. Figure 3 shows the directivity plotted with respect to the wave number $k_{b}$ in comparison to the Chu-Harrington and Geyi superdirective limits. This superdirective phenomenon is not present in PEC loops since it is necessary that the constituent material of the loop be lossy. The thin, superdirective loop antenna can be thought of as an array of two dipoles located at and directly opposite to the feed with a relative magnitude reduction of approximately $30 \%$ between the two dipoles. However, 
despite similar performance to such an array, the nanoloop does not suffer from the same drawbacks (e.g., sensitivities to the feeding network and exact placement of array elements). Though this model is useful for understanding the thin loop, the high directivity mode of the thick loop has a different physical explanation. Regardless, the thick nanoloop provides a robust, broadband gain nanoantenna with a simple, fabrication friendly geometry. A further discussion of this material involving spherical multipole decomposition, comparison to other nanoantennas, and the effect of a substrate on the radiation pattern can be found in [13].

\section{Optimization of Loop Antennas}

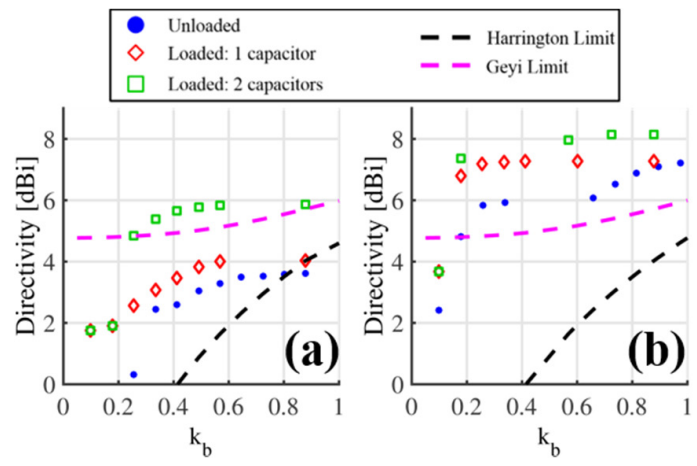

Figure 4. Pareto fronts for a) the directivity in the $(\theta, \phi)=\left(90^{\circ}, 90^{\circ}\right)$ direction and b) directivity in the $(\theta, \phi)=\left(90^{\circ}, 180^{\circ}\right)$ direction.

Given the analytical form of the theory, optimizations of dispersive nanoloops have now become highly tractable. For example, a discrete frequency sweep with 51 points can take approximately 2 hours using the full-wave solver FEKO [14] with 10 cores, while the theory implemented in MATLAB [15] with a single core takes only 20 seconds to evaluate. As mentioned in the previous section, the superdirective radiation modes of a nanoloop only manifest in the endfire direction. A reasonable question to consider is whether it is possible to redirect this mode towards a different direction. A recent study in [9] has shown that by adding capacitors, the loop may radiate superdirectively in different directions. The full Pareto fronts of the directivity at different angles versus frequency with various loading arrangements are shown in Figure 4. It is interesting that superdirectivity in the $\left(90^{\circ}, 90^{\circ}\right)$ direction can only be obtained with the application of at least two capacitors. Upon further investigation, it is determined that the only way to achieve the superdirectivity is by increasing the magnitude of the $\mathrm{m}=-2$ current mode. Similarly, increasing the magnitude of the $\mathrm{m}=+2$ current mode directs the main beam towards the $\left(90^{\circ}, 270^{\circ}\right)$ direction. From Figure $4 b$, it is notable that the nanoloop may be made to radiate with an even higher directivity in the $\left(90^{\circ}, 180^{\circ}\right)$ direction by adding capacitive loads. Additional applications of optimizations to the study and design of nanoloops can be found in [9, 16-17].

\section{References}

1. A. F. McKinley, T. P. White, I. S. Maksymov, and K. R. Catchpole, "The analytical basis for the resonances and anti-resonances of loop antennas and meta-material ring resonators," J. Appl. Phys, 112, 9, 2012, p. 094911.

2. A. E. Krasnok, I. S. Maksymov, A. I. Denisuk, P. A. Belov, A. E. Miroshnichenko, C. R. Simovski, and Y. S. Kivshar, "Optical antennas," Physics, 56, 6, 2013, pp. 539564.

3. J. E. Storer, "Impedance of thin-wire loop antennas," Trans. of the American Institute of Electrical Engineers, Part I: Communication and Electronics, 75, 5, 1956, pp. 606-619.

4. T. T. Wu, "Theory of the thin circular loop antenna," Journal of Mathematical Physics, 3, 6, 1962, pp. 13011304.

5. D. H. Werner, "An exact integration procedure for vector potentials of thin circular loop antennas," IEEE Trans. Ant. and Prop., 44, 1996, pp. 157-165.

6. A. F. McKinley, T. P. White, and K. R. Catchpole, "Theory of the circular closed loop antenna in the terahertz, infrared and optical regions," J. Appl. Phys., 114, 4, 2013, p. 044317.

7. B. Q. Lu, J. Nagar, T. Yue, M. F. Pantoja, and D. H. Werner, "Closed-form expressions for the radiation properties of nanoloops in the terahertz, infrared and optical regimes," IEEE Trans. Ant. and Prop., 65, 1, 2017, pp. 121-133.

8. A. F. McKinley, "Theory of impedance loaded loop antennas and nanorings from RF to optical wavelengths," IEEE Trans. Ant. and Prop., 65, 5, 2017, pp. 2276-2281.

9. J. Nagar, R. J. Chaky, M. F. Pantoja, A. F. McKinley, and D. H. Werner, "Optimization of far-field radiation from impedance-loaded nanoloops accelerated by an exact analytical formulation," IEEE Trans. Ant. and Prop., Under review.

10. R. Harrington and J. Mautz, "Straight wires with arbitrary excitation and loading," IEEE Trans. Ant. and Prop, 15, 4, 1967, pp. 502-515.

11. J. Nagar, B. Q. Lu, M. F. Pantoja, and D. H. Werner, "Analytical expressions for the mutual coupling of loop antennas valid from the RF to optical regimes," IEEE Trans. Ant. And Prop., 65, 12, 2017, pp, 6889-6903.

12. C. A. Balanis, Antenna Theory. New York: Wiley, 1997. 
13. M. F. Pantoja, J. Nagar, B. Lu, and D. H. Werner, "Existence of superdirective radiation modes in thin-wire nanoloops," ACS Photonics, 4, 3, 2017, pp. 509-516.

14. FEKO Suite 7.0, EM Software \& Systems - S.A., Stellenbosch, South Africa, http://www.feko.info

15. MATLAB R2014A, Mathworks - Natick, MA, USA, https://www.mathworks.com/

16. J. Nagar, S. D. Campbell, P. L. Werner, and D. H. Werner, "Multi-objective tradeoff studies of directivity achievable by electrically small nanoloops," XXXII URSI General Assembly \& Scientific Symposium, Montreal, Quebec, Canada, August 19 - August 26, 2017.

17. J. Nagar, S. D. Campbell, Q. Ren, J. A. Easum, R. P. Jenkins, and D. H. Werner, "Multi-objective optimization aided metamaterials-by-design with application to highly directive nano-devices," IEEE Journal on Multiscale and Multiphysics Computational Techniques, 2, 1, 2017, pp. 147-158. 